Available online at GSC Online Press Directory

GSC Biological and Pharmaceutical Sciences

e-ISSN: 2581-3250, CODEN (USA): GBPSC2

Journal homepage: https://www.gsconlinepress.com/journals/gscbps

(CASE REPORT)

\title{
An audit on the management of patients with SIADH (Syndrome of inappropriate ADH secretion) in a District General hospital in the UK
}

\author{
Talapatra Indrajit \\ Department of Endocrinology, Royal Albert Edward Infirmary, Wigan, UK.
}

Publication history: Received on 03 December 2019; revised on 15 December 2019; accepted on 17 December 2019

Article DOI: https://doi.org/10.30574/gscbps.2019.9.3.0230

\begin{abstract}
Syndrome of inappropriate ADH (SIADH) secretion is a condition where there is an increase in the secretion of ADH resulting in retention of excess water in the body. ADH or Antidiuretic hormone (also called Vasopressin) is produced in the hypothalamus of the brain and released by the pituitary gland. The main biochemical abnormality is low plasma sodium or hyponatraemia. However hyponatraemia can occur for multiple reasons and it is important to diagnose the specific cause as the management can vary. SIADH is a cause for euvolaemic hyponatraemia and the management includes stopping the drug responsible for causing it, fluid restriction and using medications like Tolvaptan and Demeclocycline. The audit below is an attempt to find out if the prevailing Guidelines were considered during the management of SIADH in our hospital.
\end{abstract}

Keywords: Syndrome of inappropriate ADH secretion; Euvolaemia; Hyponatraemia; Tolvaptan; Demeclocycline

\section{Introduction}

Euvolaemic hyponatraemia is the commonest cause of hyponatraemia in hospitalized patients. SIADH has to be carefully diagnosed and managed as hypotonic fluid replacement following surgery or bladder irrigations with hypotonic saline following transurethral prostate resection in hospital can also lead to euvolaemic hyponatraemia [1, 2]. Also ACTH (Adrenocorticotrophic hormone) deficiency, which also causes euvolaemic hyponatraemia, needs to be excluded before diagnosing SIADH [3]. The symptoms and the initial assessment of hyponatraemia have been discussed below.

The criteria for diagnosing SIADH include

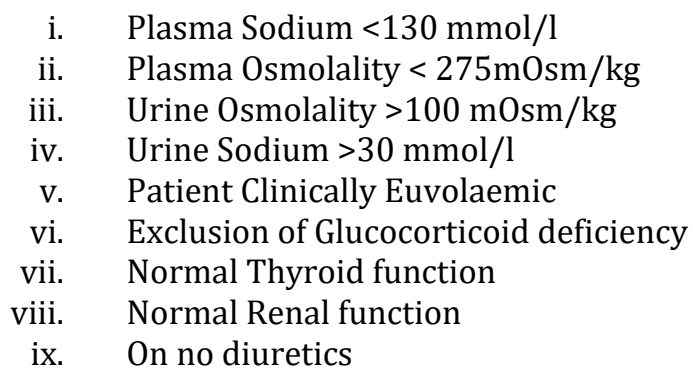

The objective of the audit was to check if patients with actual diagnosis of SIADH were referred to the Endocrinology unit and if such patients were managed according to the current Guidelines.

\footnotetext{
${ }^{*}$ Corresponding author

E-mail address: indra_tala@hotmail.com
} 


\section{Aim of the Audit}

To find out if the patients seen for hyponatraemia and diagnosed as SIADH (including Ward Referrals and OPD Cases) between April and July 2019 were managed in accordance with the prevailing Guidelines.

\section{Methods}

Patient seen from April 2019 for 4 months both on the Wards and the OPD (Referrals) were audited for the purpose. Patients seen by Registrar, Associate Specialist and the different Consultants were studied for the purpose of the Audit.

\subsection{Guidelines}

The European Guidelines were followed for the purpose of the audit.

\subsection{Criteria of the audit}

i. To find out if the hydration status was assessed and documented.

ii. To find out if appropriate referrals were made to Endocrinology. We expect referrals in cases of euvolaemic hyponatraemia.

iii. To find out if before referral TFTs and an early morning Cortisol were done.

iv. To find out if before referral plasma osmolality, urinary osmolality and urine Na were done

v. To find out if the offending agent, if any, was stopped

vi. To find out if appropriate imaging were done like CXR (Chest X-ray), CT TAP (Thorax, Abdomen and Pelvis), CT head

vii. Whether the patient was put on fluid restriction (500-1000 ml/24 hours) prior to referral

viii. Whether Tolvaptan was used and if so what initial dose was used

ix. Whether fluid restriction was removed during use of Tolvaptan

x. If Plasma sodium was checked at 6, 12, 18 and 24 hours after initial Tolvaptan dose

xi. If rise of Plasma sodium in 24 and 48 hours following administration of Tolvaptan was documented

xii. Whether Demeclocycine was used

xiii. Whether appropriate referral was made to ITU.

\subsection{Symptoms of mild to moderate of hyponatraemia}

Headache, fatigue, lack of concentration, low mood, memory impairment, nausea, gait disturbance and falls and muscle cramps.

\subsection{Advanced symptoms of hyponatraemia (with Plasma $\mathrm{Na}<120 \mathrm{mmol} / \mathrm{l}$ )}

Confusion, somnolence, vomiting, hallucinations, acute psychosis, coma.

\subsection{Initial assessment of hyponatraemia}

\section{(For management of hyponatraemia at a glance refer to Appendix 1)}

\begin{tabular}{|c|c|c|}
\hline \multicolumn{3}{|c|}{ Assessment of the hydration status of the patient } \\
\hline & $\downarrow$ & \\
\hline Hypovolaemic & Euvolaemic & Hypervolaemic \\
\hline (Common causes: Diarrhoea, & Urine $\mathrm{Na}<30$ (Hypothyroidism) & Cirrhosis, CCF \\
\hline Vomiting, Diuretics, Burns) & Urine $\mathrm{Na}>30($ SIADH, low ACTH $)$ & Nephrotic syndrome. \\
\hline
\end{tabular}

To Check Thyroid function tests (TFTs) and early morning Plasma Cortisol. If Plasma Cortisol is low, perform a SST (Short Synacthen Test). 


\author{
Management of SIADH (once diagnosis is established) Ref: [4-6] \\ $\downarrow$ \\ Restrict Fluids to 500-1000 ml of water daily \\ $\downarrow$ \\ Investigate and treat the cause (CXR, CT TAP, CT Head, stop drugs known to cause low sodium) \\ $\downarrow$ \\ Referral to Endocrinology team \\ $\downarrow$ \\ Tolvaptan from $7.5 \mathrm{mg}$ to $15 \mathrm{mg}$ daily initially. \\ Measure plasma sodium at 6,12,18 and 24 hours. \\ Patient allowed free fluids. The dose thereafter is adjusted according to the response \\ (Tolvaptan is the drug of choice if treatment is commenced on the ward) \\ Monitor LFTs and stop it if patient develops features of hepatic impairment.
}

(Demeclocycline 600-1200 mg, with monitoring of kidney function and for skin photosensitivity, is the drug of choice if treatment is commenced at the out-patients)

$\downarrow$

Aim to increase plasma sodium by a maximum of $10 \mathrm{mmol} / \mathrm{l}$ over 24 hours and $18 \mathrm{mmol} / \mathrm{l}$ over 48 hours

$\downarrow$

If the patient is acutely symptomatic and plasma sodium is not improving, refer to ITU for treatment with hypertonic saline.

$2.7 \%$ or $3 \%$ saline, if available, can be administered with/without loop diuretics at the discretion of the ITU Consultant

On admission patients with acute symptomatic hyponatraemia (with plasma sodium of $>115 \mathrm{mmol} / \mathrm{l}$ ), or if the plasma sodium is $<115 \mathrm{mmol} / \mathrm{l}$, should be discussed with ITU

Follow up of patients discharged on Tolvaptan is required at the out-patient department once every one or two weeks initially depending on the response of the patient.

\title{
4. Results
}

i. Total No of Patients $=21$

ii. Male: Female 8:13;

iii. Age in years $40-49=1,50-59=1,60-69=3,70-79=5,80-89=8,>90=3$

iv. Ward Referral 15 (71\%); GP Referral 6 (21\%)

v. Hydration status was assessed and documented $=5 / 21(24 \%)$

vi. If before referral TFTs and an early morning Cortisol were done: Cortisol done in $17 / 21$ or $81 \%$ (not done in 3 patients referred by GPs; not done in 1 patient referred from another Ward); TFTs done in 20/21 $(95 \%)$

vii. If before referral plasma osmolality was done: 19/21 (90\%); urinary osmolality was done: 15/21 (71\%) and urine sodium done: $14 / 21(67 \%)$

viii. The offending agent if any (diuretics, antipsychotics, ACE-1, PPI), was stopped: 6/21 (29\%) of patients were on medications capable of causing hyponatraemia. In 5/6 (84\%) patients the drugs were stopped

ix. Appropriate imaging were done before referral like CXR (21/21 or 100\%), CT TAP and CT head (13/21 or $62 \%)$

$\mathrm{x}$. Whether the patient was put on fluid restriction (500-1000 ml/24 hours) prior to referral (14/21 or $67 \%$; $14 / 15$ Ward patients were on fluid restriction); however $3 / 14$ were on $1.5 \mathrm{~L} / 24 \mathrm{hrs}$ and $1 \mathrm{on} 1.2 \mathrm{~L} / 24 \mathrm{hrs}$ )

xi. Tolvaptan used; 5/15 ward patients (33\%); Dose (4/5 or $80 \%-1^{\text {st }}$ dose-7.5 mg; $1 / 5$ or $20 \%-1$ st dose 15 $\mathrm{mg}$ ) 
xii. $\quad$ Fluid restriction removed during use of Tolvaptan $(3 / 5$ or $60 \%)$

xiii. Plasma Na checked at 6, 12, 18 and 24 hours after initial Tolvaptan dose: 5/5 (100\%)- at least 2 or 3 times over 24 hours

xiv. Rise of Plasma Na in 24 and 48 hours following administration of Tolvaptan documented: 5/5 or 100\%

$\mathrm{xv}$. Whether Demeclocycine was used: 1 from OPD

xvi. Appropriate referral was needed and made to ITU: none

\subsection{Results of audit interpreted in a Graphical manner}

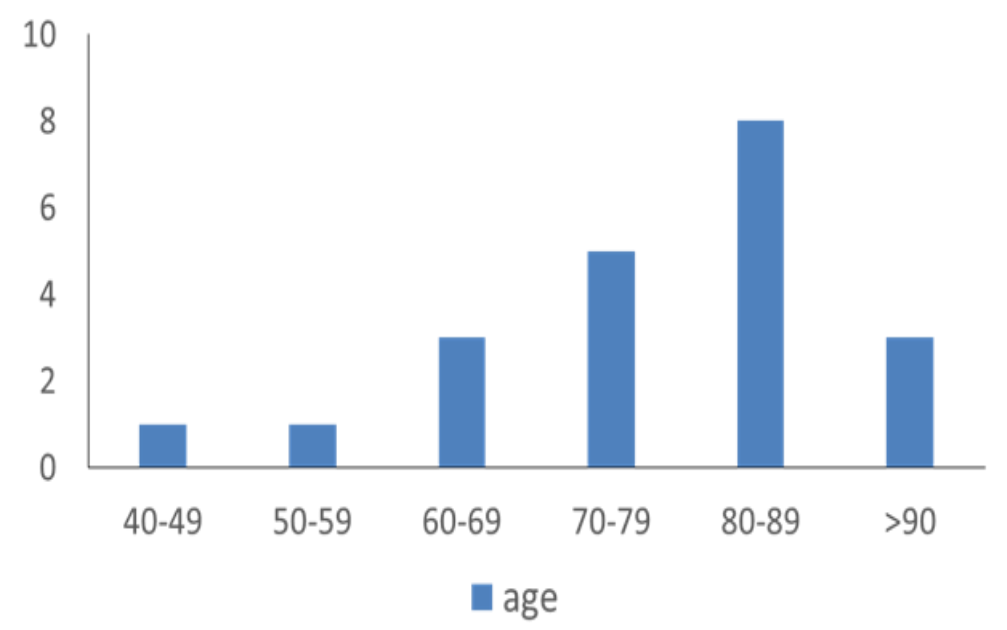

Figure 1 Bar diagram showing age distribution of patients

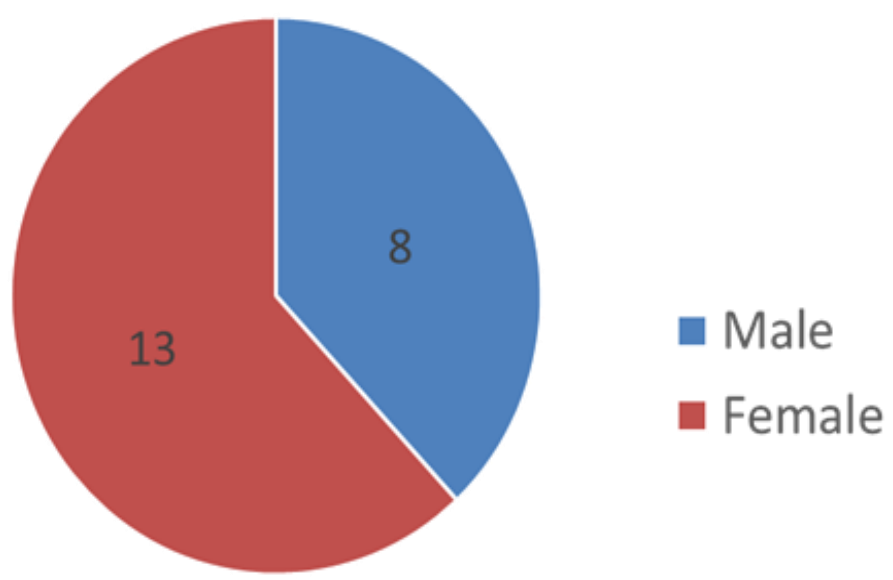

Figure 2 Pie chart showing gender distribution of patients 
Talapatra / GSC Biological and Pharmaceutical Sciences 2019, 09(03), 091-098

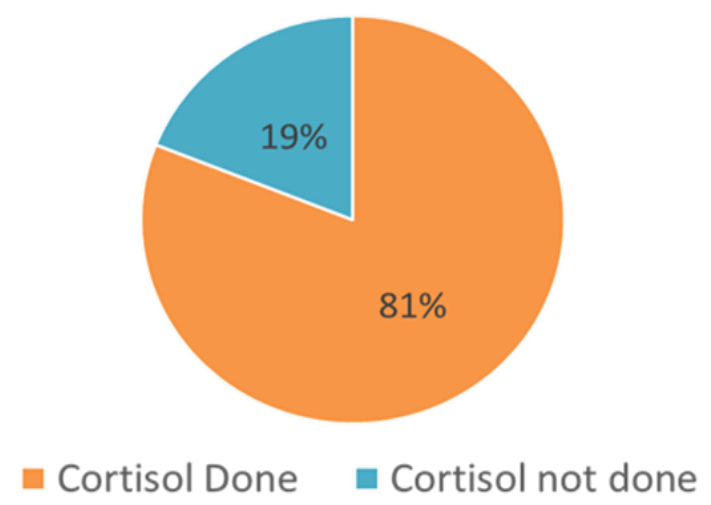

Figure 3 Pie chart showing percentage of patients who had plasma cortisol level done

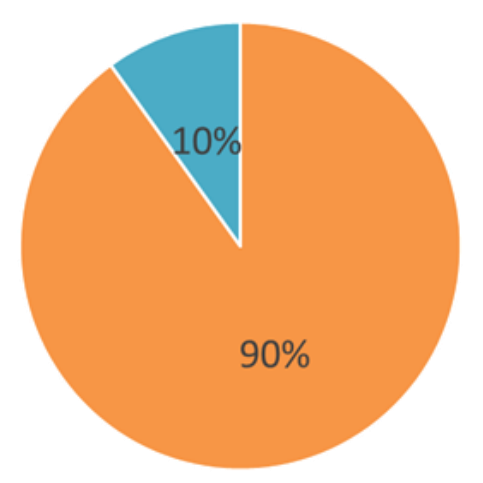

- Done Not done

Figure 4 Pic chart showing percentage of patients who had plasma osmolality done.

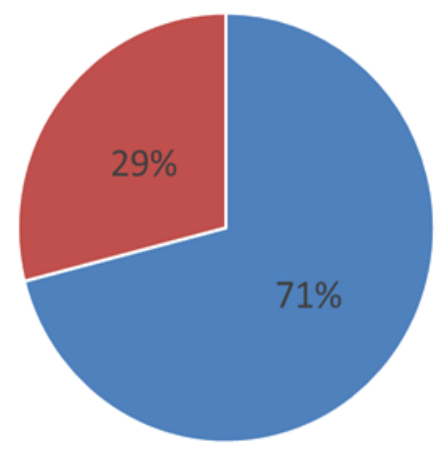

- Done Not done

Figure 5 Pie chart showing percentage of patients who had urine osmolality done 
Talapatra / GSC Biological and Pharmaceutical Sciences 2019, 09(03), 091-098

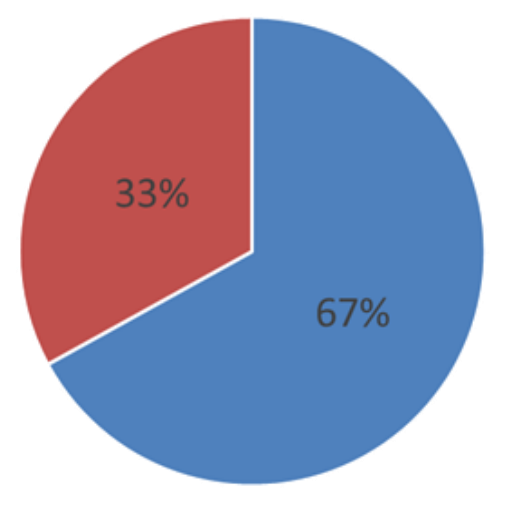

- Done not Done

Figure 6 Pie chart showing percentage of patients who had urine sodium done
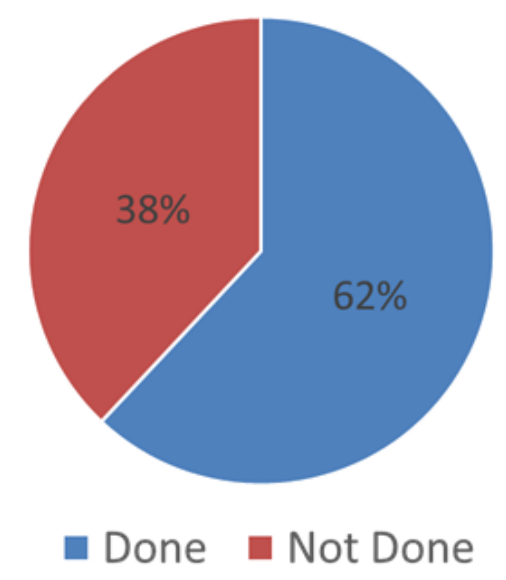

Figure 7 Pie chart showing percentage of patients who had CT Head and CT TAP done

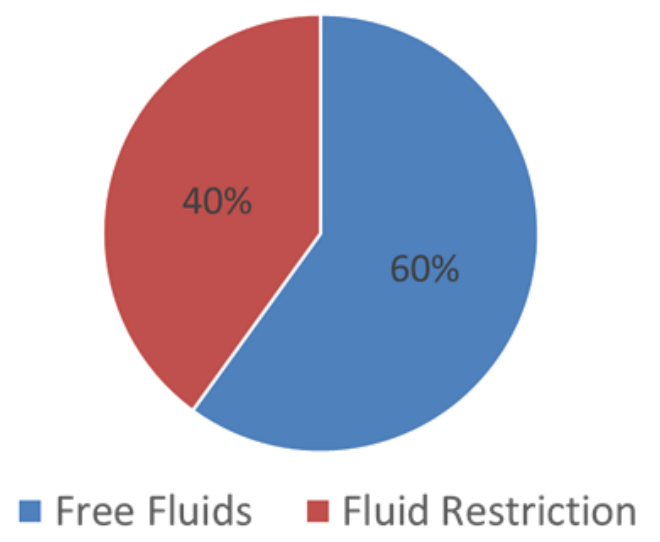

Figure 8 Pie chart showing percentage of patients allowed free fluids while on Tolvaptan 


\section{Conclusion}

The hydration status was documented in 5 out of 15 ward patients which needs to be looked into. The morning Cortisol was measured in $81 \%$ patients which was quite good. The Urine Osmolality was measured in $71 \%$ patients and Urine sodium in $67 \%$ cases and both need to be improved. There was a history of intake of potential hyponatraemic medications in 6 cases; in 5 cases the drugs were stopped. In one patient the anti-psychotic medication could not be stopped. In the audit CT TAP/Head were found to be done in $62 \%$ cases. There is a definite room for improvement as an undetected malignancy or a CNS cause can lead to the development of SIADH. On the Wards 14/15 patients were put on fluid restriction which was a very good performance; however 10 out of 14 patients were on $1 \mathrm{~L}$ or less of fluids over 24 hours. Free Fluids were allowed only in $60 \%$ of patients put on Tolvaptan. Ideally to prevent rapid rise of Plasma sodium and central pontine demyelination, all patients on Tolvaptan should be allowed free fluid intake. Plasma sodium measurement within 24 hours after first dose of Tolvaptan was satisfactory and documentation of Plasma sodium 24 and 48 hours after initiating Tolvaptan (Rise in Plasma sodium not to exceed $10 \mathrm{mmol} / \mathrm{l}$ and $18 \mathrm{mmol} / \mathrm{l}$ respectively) was done in all cases.

\section{Recommendations}

- Hydration status should be documented in all cases; euvolaemic hyponatraemia occurs in SIADH

- Early morning Cortisol and TFTs are to be checked in all cases before referral to Endocrine unit

- Paired Plasma and Urine Osmolality and Urine sodium should be checked in all patients before referral

- Any offending drug should be stopped, if possible, before referral

- Proper imaging should be done; CT TAP is needed (to detect malignancy) before referral

- $\quad$ Fluid restriction should be between 500-1000 ml over 24 hours

- Fluid restriction is to be removed if the patient is put on Tolvaptan

- Plasma Sodium is to be measured at 6,12,18 and 24 hours following initial administration of Tolvaptan

- To ensure that Plasma sodium does not rise by more than $10 \mathrm{mmol} / \mathrm{l}$ in 24 hours and $18 \mathrm{mmol} / \mathrm{l}$ after 48 hours following administration of Tolvaptan

- Demeclocycline remains the drug of choice from the OPD

- If the GPs (General Practitioners) are to be informed to do the preliminary investigations (TFTs, Cortisol, Paired Plasma and Urine Osmolality, Urine sodium and imaging) prior to referral of patients with low Plasma sodium, needs to be discussed in Endocrinology meeting

- Aim to re-audit in 1 year.

\section{Appendix}

\subsection{Hypovolaemic Hyponatraemia}

(Reduced skin turgor, tachycardia, low BP, postural hypotension): Treat with $0.9 \% \mathrm{~N}$ saline.

\subsection{Hypervolaemic Hyponatraemia}

(oedema, raised JVP, LVF, Ascitis): Treat the underlying cause like Cardiac failure, Renal failure, Liver cirrhosis.

\subsection{Euvolaemic Hyponatraemia with diagnosis of SIADH}

Fluid restriction and follow the Guideline discussed

For fluid restriction the following regime can be considered

Calculate electrolyte free water clearance using Furst Formula: (Urine $\mathrm{Na}+\mathrm{K}$ )/ Serum $\mathrm{Na}$

$<0.5$ - Commence $1 \mathrm{~L} / \mathrm{d}$ fluid restriction

0.5-1-Commence $0.5 \mathrm{~L} / \mathrm{d}$ fluid restriction

$>1$ - Fluid restriction not advised. 


\section{Compliance with ethical standards}

\section{Acknowledgments}

I like to acknowledge the Endocrine secretaries for providing the required list of patients for the purpose of the audit.

\section{References}

[1] Hahn RG. (2006). Fluid absorption in endoscopic surgery. British Journal of Anaesthesia, 96 (1), 8-20.

[2] Osborn DE, Rao PN, Greene MJ and Barnard RJ. (1980). Fluid absorption during transurethral resection. British Medical Journal, 281 (6254), 1549-1550.

[3] Oelkers W. (1989). Hyponatremia and inappropriate secretion of vasopressin (antidiuretic hormone) in patients with hypopituitarism. New England Journal of Medicine, 321, 492-496.

[4] Gross P. (2012). Clinical management of SIADH. Therapeutic Advances in Endocrinology and Metabolism, 3 (2), 61-73.

[5] Spasovski G, Vanholder R, Allolio B, Annane D, Ball S, Bichet D et al. (2014). Clinical practice guideline on diagnosis and treatment of hyponatraemia. European Journal of Endocrinology, 170 (3), G1-47.

[6] Grant p, Ayuk J, Bouloux PM, Cohen M, Cranston I, Murray RD, Rees A, Thatcher N and Grossman A. (2015). The diagnosis and management of inpatient hyponatraemia and SIADH. European Journal of Clinical Investigation, 45 (8), 888-894.

\section{How to cite this article}

Talapatra I. (2019). An audit on the management of patients with SIADH (Syndrome of inappropriate ADH secretion) in a District General hospital in the UK. GSC Biological and Pharmaceutical Sciences, 9(3), 91-98. 